\title{
Dynamic neural networks for real-time water level predictions of sewerage systems-covering gauged and ungauged sites
}

\author{
Yen-Ming Chiang ${ }^{1}$, Li-Chiu Chang ${ }^{2}$, Meng-Jung Tsai ${ }^{1}$, Yi-Fung Wang ${ }^{3}$, and Fi-John Chang ${ }^{1}$ \\ ${ }^{1}$ Department of Bioenvironmental Systems Engineering, National Taiwan University, Taipei, Taiwan \\ ${ }^{2}$ Department of Water Resources and Environmental Engineering, Tamkang University, Taipei, Taiwan \\ ${ }^{3}$ Water Resources Agency, Ministry of Economic Affairs, Taipei, Taiwan
}

Received: 17 March 2010 - Published in Hydrol. Earth Syst. Sci. Discuss.: 13 April 2010

Revised: 27 June 2010 - Accepted: 6 July 2010 - Published: 16 July 2010

\begin{abstract}
In this research, we propose recurrent neural networks (RNNs) to build a relationship between rainfalls and water level patterns of an urban sewerage system based on historical torrential rain/storm events. The RNN allows signals to propagate in both forward and backward directions, which offers the network dynamic memories. Besides, the information at the current time-step with a feedback operation can yield a time-delay unit that provides internal input information at the next time-step to effectively deal with time-varying systems. The RNN is implemented at both gauged and ungauged sites for 5-, 10-, 15-, and 20-min-ahead water level predictions. The results show that the RNN is capable of learning the nonlinear sewerage system and producing satisfactory predictions at the gauged sites. Concerning the ungauged sites, there are no historical data of water level to support prediction. In order to overcome such problem, a set of synthetic data, generated from a storm water management model (SWMM) under cautious verification process of applicability based on the data from nearby gauging stations, are introduced as the learning target to the training procedure of the RNN and moreover evaluating the performance of the RNN at the ungauged sites. The results demonstrate that the potential role of the SWMM coupled with nearby rainfall and water level information can be of great use in enhancing the capability of the RNN at the ungauged sites. Hence we can conclude that the RNN is an effective and suitable model for successfully predicting the water levels at both gauged and ungauged sites in urban sewerage systems.
\end{abstract}

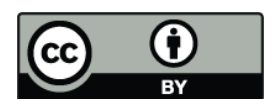

Correspondence to: F.-J. Chang (changfj@ntu.edu.tw)

\section{Introduction}

The growth of urbanization has paralleled the growth of the global economy over the last decades. Owing to the rapid development of metropolitan areas, the natural hydrological mechanisms have been changed, such as the reduction of the infiltration and the concentration response time in a catchment, which leads to unexpected inundations and failures in operating pumping stations. Taiwan is prone to such circumstances. Taiwan is located in the northwestern Pacific Ocean where the activities of subtropical air currents happen frequently. Due to the irregular timing and location of precipitations and the increase of impervious areas, the flood hydrographs during typhoon seasons result in large peak flows with fast-rising limbs which usually cause serious disasters in Taiwan. For example, on 17 September 2001, Typhoon Nari struck northern Taiwan accompanied with heavy rainfalls, more than $500 \mathrm{~mm} /$ day, which caused 27 deaths. The flood inundated 4151 building basements that brought on countless economic losses.

A surface inundation will occur as the surface runoff discharge is larger than the designed capacity of a storm drainage system. The operation of a pumping station highly depends on the information of water level. The risks of inundations may be reduced if accurate water level prediction information can be provided. With such prediction information, a peak flow can be mitigated by pumping out the inner water prior to the approach to a peak flow. Despite of the massive researches that have been invested in simulating the water levels of sewerage systems in storm events, the time-delay and the magnitude of a peak flow still can not be estimated precisely and a comprehensive solution of the assessment of sewerage systems does not seem to be on hand, either. In present practice, the assessments of the water level variations in sewerage systems during storm events count on

Published by Copernicus Publications on behalf of the European Geosciences Union. 
conceptual models mainly. To implement these conceptual models, it requires data generated from sewerage systems, e.g. storage volume, pumping capacity, contribution areas, and so on, and hydraulic loads such as precipitations and flows, etc. The conceptual models can only provide simulation results on the basis of given rainfall conditions and are not commonly used as real-time operation tools. For quantifying the influences of water level variations in sewerage systems and increasing the operation ability of pumping stations, this research focuses on developing real-time models to produce accurate multi-step-ahead water level predictions for urban sewerage systems by utilizing recurrent neural networks (RNNs).

The artificial neural network (ANN) has evolved as a branch of artificial intelligence and has been regarded as an efficient tool for the learning of any nonlinear inputoutput systems. Therefore, the ANN is described as a data processing system that composed of many nonlinear interconnected artificial neurons. The main benefit of adapting ANNs are that they can effectively extract significant features and trends from complex data structures even if the underlying physics is either unknown or difficult to recognize. In the field of hydrology, various research results that were produced by the ANNs have reported the improvements in the performances of simulations such as rainfall forecasting (Chiang and Chang, 2009; Chiang et al., 2007a; Hung et al., 2009; Nasseri et al., 2008), reservoir operation (Chandramouli and Deka, 2005; Chang and Chang, 2001; Chaves and Chang, 2008), stream flow forecasting (Akhtar et al., 2009; Chang and Chang, 2006; Chiang et al., 2007b; Maity and Kumar, 2008; Sudheer et al., 2008; Toth, 2009; Sahoo et al., 2009; Besaw et al., 2010), and applications in urban drainage systems (Bruen and Yang, 2006; Loke et al., 1997).

In viewing the network topologies and the structures of the ANNs used in the field of hydrology, we can distinguish them into two different generic neural network types: feedforward and feedback networks. The topology of the feedforward ANN consists of a set of neurons connected by the links in a number of layers. The feedforward networks implanted fixed-weights which map the input space to the output space, so that the state of any neuron is solely determined by the input-output pattern excluding the initial and the past states of the neuron. That is to say, the feedforward networks are not dynamic. The corresponding advantage is that a feedfoward network can be built easily by a simple optimization algorithm. For such reason, this type of network architecture is the most popular in use today. Nonetheless, the static feedforward neural network also has several drawbacks for some applications. As for the feedback architecture, it differentiates itself from the feedforward one by possessing at least one feedback link. The presence of a feedback link has a profound impact on the learning capability of the RNN and on its performance (Coulibaly and Baldwin, 2005). Because of the feedback link, the status of its neuron depends not only on the current input signal, but also on the previous states of the neuron. The chief advantage of the dynamic feedback neural network is that it can effectively decrease the input dimension of the network and therefore improve the training efficiency (Chang et al., 2002; Chiang et al., 2004). In this research, we propose a RNN for water level predictions in sewerage systems. There are two main features of the RNN; (1) its nonlinear properties make the prediction of a complex nonlinear dynamic system feasible, and (2) its temporal recurrent processing properties make the implementation easier.

Due to the limited number of water level gauging stations in a sewerage system, the data and predictions of water levels at ungauged sites become an important issue of flood prevention. One of the advantages of predicting water levels at ungauged sites is to reduce the expensive engineering and maintenance costs. In view of this, the storm water management model (SWMM), a well-known tool for modeling urban water circulations, is introduced in this research. A SWMM is an urban runoff model designed for simulating the quantity and quality of flows associated with urban surface runoffs and combined with sewer overflow phenomena (Huber and Dickinson, 1988). A SWMM could provide hydrographs at any manhole and give conceptual flood depths by water levels, and therefore was adapted for simulating and generating data of water levels at an ungauged site. Its synthetic data, under certain verification process of applicability based on the data from nearby gauging stations, were further used as the learning targets to the training procedure of the RNNbased hydrological models and also used for evaluating and verifying the performance of the RNNs.

The difference between RNN and SWMM is that, in general, a physically based simulation model such as SWMM can be used to model natural systems to gain insight into their functioning and to show the eventual real effects of alternative conditions. The SWMM model could generate the water level of time $t$ by being fed with input data at the same time (time $t$ ). In this study, the calibrated SWMM model was fed with the whole series of input information to gain its corresponding series of estimation outputs. Consequently, it can only produce current water level because future rainfall pattern is not available at current time. Whereas the trained RNN can produce future water level (time $t+n$ ) by being fed with current data (time $t$ ) and the model can be executed through a step by step procedure. Concretely speaking, the output of SWMM is suitable for hydraulic planning and design after a storm event because the model requires complete rainfall pattern of an event; whereas the output of RNN is suitable for real-time operation of pumps because the model can be executed through a step by step procedure.

In this research, we briefly illustrate both RNN and SWMM methods at first. And next, the study area and the structure of water level prediction models are presented. Results obtained from both gauged and ungauged sites are analyzed in the subsequent sections, and conclusions are drawn in the end. 


\section{Methodology}

\subsection{Recurrent neural network (RNN)}

Many types of artificial neural networks have been developed in the last few decades, which mostly belong to feedforward operations, so-called static neural networks, in spite of different architectures of these networks. The static feedforward neural network can achieve satisfactory analytical outcomes if sufficient data are available (Chiang et al., 2004). Nevertheless, in consideration of the temporal or short-term dynamic property, the static neural network has difficulty in recognizing and predicting the reality. The definition of a RNN, so-called dynamic neural network, is that at least one feedback link should be added to the static neural network. The RNN allows signals to propagate in both forward and backward directions, which offers the network dynamic memories. Besides, the information at the current time-step with a feedback operation can yield a time-delay unit that provides internal input information at the next time-step. In other words, the recurrent neural networks are able to capture the true hidden dynamic memories of nonlinear systems. The RNN has been proved to be a powerful method for handling complicated systems such as nonlinear time-varying systems (Coulibaly and Baldwin, 2005; Coulibaly and Evora, 2007; Kumar et al., 2004; Mishra and Desai, 2006; Razavi and Karamouz, 2007; Yazdani et al., 2009).

In this research, we propose a three-layer RNN with internal time-delay feedback loops in both hidden and output layers, see Fig. 1. The transfer function of both hidden and output layers is of sigmoid type which can be applied to nonlinear transformation. Each input neuron is connected to a hidden neuron, where each hidden neuron has its corresponding time-delay unit, i.e. the number of hidden neurons is equal to that of time-delay units. Basically, a feedback link allows its time-delay unit to store the output information of this hidden neuron as an additional input to all hidden neurons at next time-step, refer to Fig. 5 for details. Then, the RNN has an inherent dynamic memory given by the feedback connections of the time-delay units, and its output depends not only on the current input information but also on the previous states of the network. In this research, the RNN makes use of the gradient descent method for calibrating the parameters via minimizing the forecasting errors.

As shown in Fig. 1, the output of neuron $J$ in the hidden layer is computed as

$y_{J}(t+1)=f\left(\sum_{i=1}^{I} w_{J i} X_{i}(t)+\sum_{j=1}^{J} w_{J j} y_{j}(t)\right)$

where $f($.$) denotes the transfer function, y_{j}(t)$ denotes the output of the hidden neuron $J$ at time $t, w_{J i}$ denotes the connection weight from the input neuron $i$ to the hidden layer neuron $J, X_{i}(t)$ denotes the input, and $w_{I j}$ denotes the timedelay feedback weight from the hidden neuron $j$ to the hidden neuron $J$.

\section{Outputs}

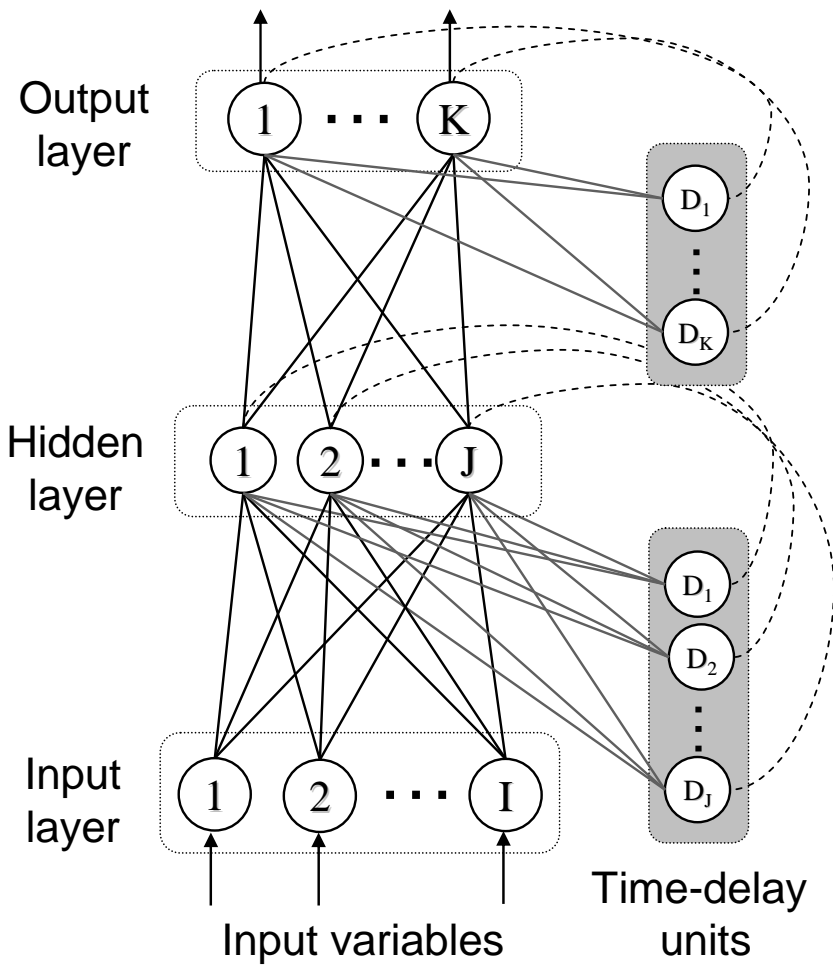

Fig. 1. The architecture of the recurrent neural network (RNN).

The output of neuron $K$ in the output layer at time $t+1$ is calculated by

$\hat{Q}_{K}(t+1)=f\left(\sum_{j=1}^{J} w_{K j} y_{j}(t+1)+\sum_{k=1}^{K} w_{K k} \hat{Q}_{K}(t)\right)$

where $w_{K j}$ represents the connection weight from the hidden unit $j$ to the output unit $K$, and $w_{K k}$ represents the time-delay feedback weight from the output unit $k$ to the output unit $K$.

The associated weight of each parameter in the RNN can be figured out by the following formula through the chain rule with partial derivatives.

$W_{\text {new }}=W_{\text {old }}-\eta \frac{\partial E_{\text {total }}}{\partial W}$

where $\eta$ denotes the learning rate.

$E_{\text {total }}$ represents the objective function

$E_{\text {total }}=\sum_{t=1}^{N} E(t)=\frac{1}{2} \sum_{t=1}^{N} \sum_{k=1}^{K}\left[Q_{k}(t)-\hat{Q}_{k}(t)\right]^{2}$

where $Q_{k}(t)$ represents the value of the learning target to neuron $k$ at time $t$; and $\hat{Q}_{K}(t)$ is the network output of neuron $k$ at time $t$. 


\subsection{Storm water management model (SWMM)}

The SWMM is a comprehensive hydrological simulation model that is commonly applied to designing the quality and quantity models of runoffs in urban areas (Ackerman and Schiff, 2003; Baffaut and Delleur, 1990; Borah and Bera, 2003; Denault et al., 2006; Maharjan et al., 2009; Tsihrintzis and Hamid, 1998). As understood, even though the RNN can easily build a water level prediction model at the gauged sites, the difficulty in predicting the water levels at an ungauged site is due to its unavailability of real observations for the training and evaluation of a RNN. Fortunately, the SWMM can be an appropriate tool to overcome such difficulty by its simulation ability. In principle, the SWMM is capable of simulating the quantity and quality of runoffs within a drainage basin, and thus is chosen to simulate the water levels of sewerage systems at ungauged sites. Hence, the major reason for applying a SWMM to this research is to generate synthetic data of the water levels from a specific manhole at an ungauged site. To simulate a flow, the SWMM uses the Saint-Venant equations for a gradually varied, turbulent, and unsteady flow. The Saint-Venant equations represent the principles of conservation of momentum and conservation of mass. A SWMM contains four functional program blocks that can simulate different components of a hydrological circulation. Two of them are managed herein: the RUNOFF and the EXTRAN blocks (Huber and Dickinson, 1988). The RUNOFF block performs hydrological computations according to the theory of nonlinear reservoirs, and the RUNOFF outputs are then taken as inputs to the EXTRAN block which is designed to route the flows in a sewerage system by using numerical methods.

Next, the SWMM parameters recruited in this research comprise ten factors which are catchment length/width ratio, catchment slope, maximum infiltration, minimum infiltration, impervious area Manning's roughness coefficient, pervious area Manning's roughness coefficient, impervious area detention storage, pervious area detention storage, $\%$ of impervious area of catchment, and decay rate of infiltration curve. The main reason for not using the suggested parameters listed in the table of the SWMM manual or the parameters presented in previous research papers is that, empirically, these parameters may not fit in with the analysis of the storm events occurred in Taiwan. These parameters are further calibrated by use of the historical observation values of rainfalls and runoffs. A detailed discussion is illustrated in next sections.

\section{Applications}

\subsection{Study area and data}

Taiwan is located in the subtropical jet stream monsoon district of northern Pacific Ocean. Taipei City is situated in the
Taipei Basin of northern Taiwan and is surrounded by the Danshui River, whose narrow estuary makes it difficult to discharge water effectively from the city. A highly intensive rainfall during a storm or a typhoon could easily cause flooding. The flood control strategy covers two actions; (1) to build embankments along the river sides, and (2) to set pumping stations at the main drains to discharge the rain water from the city. Pumping stations are the principal hydraulic facilities for the sluices of floods in highly developed cities, and therefore play important roles in mitigating the floods in metropolitan areas. Accurate predictions of water levels in urban drainage systems are necessary and important for successful operations of the pumping stations. The study area for this research is the Yu-Cheng catchment, located in southeastern Taipei as shown in Fig. 2, which is taken for a detailed investigation of water levels for prediction purpose. There are five rain gauging stations, denoted blue circles in Fig. 2, and ten water level gauging stations, denoted red triangles in Fig. 2, in this region. The Yu-Cheng catchment with an area about 1645 ha owns the biggest drainage system in Taipei City and the pumping station was built in 1987 to drain or pump the inner water into the Keelung River. The pumping station contains seven massive pumps and has the total capacity to $184.1 \mathrm{~cm} / \mathrm{s}$, which was the most advanced and largest in Asia in the 1980s. When the water level of the river rises up to the warning level $(1.8 \mathrm{~m})$, the pumps turn and warm up. These pumps start pumping when the water level of Keelung River is higher than start-out level $(2.4 \mathrm{~m})$; meanwhile, the gravity gates are immediately closed which means the running water cannot be discharged by gravity. The start-out level is the lowest water level for pumps functioning; the pumps may be damaged if the water is lower than this level. These seven pump sets are operated independently and sequentially according to the different water levels which means when a running pump cannot control the water level under the level of $2.4 \mathrm{~m}$, another pump will start working rather than turn on all pumps during typhoon periods. On the contrary, these pumps are turned off, respectively, as the water level is falling.

In this research, the RNN-based model was constructed for precise predictions of water levels at the gauging stations. The outlet of the sewerage system is the water level gauging station, Station YC10, and therefore Station YC10 is selected as the objective for a water level prediction. The real-time water level monitoring of the sewerage systems has been operated by the Hydraulic Engineering Office of Taipei City Government for the past few years. The historical data used in this research contained the precipitations and water level information recorded from 2002 to 2006 . The precipitation observation values collected in this research were used to calculate the mean areal precipitation based on the Thiessen polygon method in order to effectively reduce the input dimension of the RNN. After data preprocessing, a total of 2055 records of data, extracted from 14 typhoons or storm events, with a resolution of $5 \mathrm{~min}$ were collected, see Table 1. 


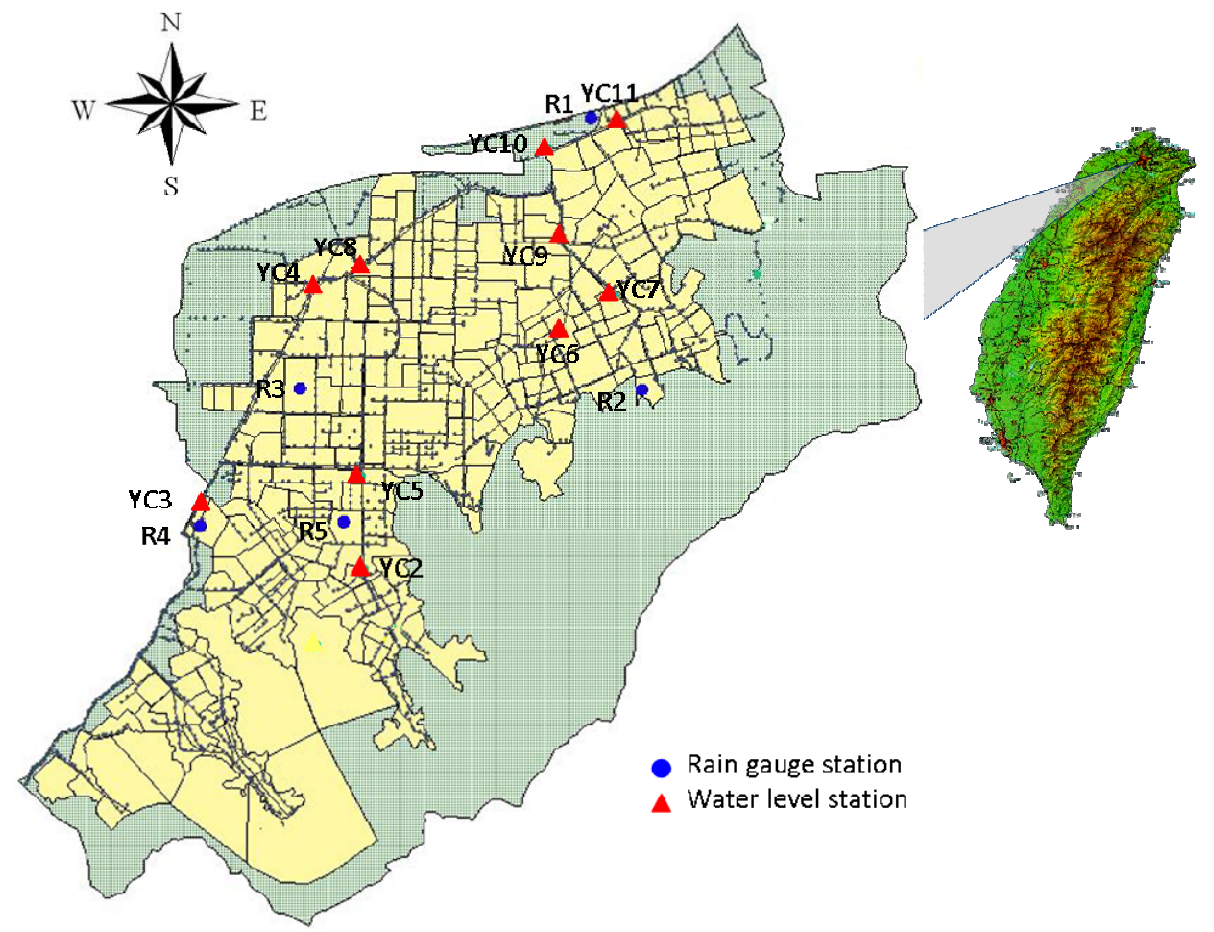

Fig. 2. The locations of Yu-Cheng catchment and the monitoring stations.

Table 1. Data from storm events for water level predictions.

\begin{tabular}{llllll}
\hline Config. & Event & Amount & $\begin{array}{l}\text { Rainfall } \\
\text { accumulation } \\
(\mathrm{mm})\end{array}$ & $\begin{array}{l}\text { Mean water } \\
\text { level } \\
(\mathrm{m})\end{array}$ & $\begin{array}{l}\text { Standard } \\
\text { deviation } \\
(\mathrm{m})\end{array}$ \\
\hline 1 & & 395 & 90.8 & 2.10 & 0.61 \\
2 & & 85 & 20.9 & 2.25 & 0.33 \\
3 & & 68 & 15.0 & 2.28 & 0.26 \\
4 & & 107 & 33.0 & 1.44 & 0.57 \\
5 & Training & 31 & 68.4 & 1.84 & 0.27 \\
6 & & 256 & 406.5 & 5.48 & 1.26 \\
7 & & 206 & 156.4 & 3.20 & 0.38 \\
8 & & 300 & 156.1 & 2.63 & 0.74 \\
\hline 9 & & 135 & 123.0 & 2.32 & 0.83 \\
10 & Validation & 60 & 61.8 & 1.77 & 0.62 \\
11 & & 43 & 21.0 & 1.33 & 0.15 \\
\hline 12 & & 142 & 53.2 & 2.06 & 0.65 \\
13 & Testing & 176 & 57.2 & 2.04 & 0.55 \\
14 & & 51 & 25.3 & 1.46 & 0.65 \\
\hline
\end{tabular}

These data were divided into three parts; (1) data associated with eight events were arranged to train the RNN parameters, (2) data associated with the other three events were dedicated to validate the RNN, and (3) data associated with the remaining three events were for the testing procedure of the RNN.

\subsection{Water level prediction model}

In this research, the RNNs were built for the water level predictions at both gauged and ungauged sites in a sewer- age system. The purpose of predicting the water level at the select gauged site is to provide upcoming information (predictions of water level) for following operation of pumps at Yu-Cheng station. For predicting the water level of Station $\mathrm{YC} 10$, the input information to the RNN-based hydrological model mostly came from the upstream water levels and the mean areal precipitations. In addition to the mean areal precipitation information, the variation of the water level at the upstream gauging station may also affect the water level at Station YC10 if the upstream gauging station is highly correlated to Station YC10. On the basis of the main drains of the watershed, the drainage system of the Yu-Cheng catchment can be roughly partitioned into three sub-drainage systems. The outlets of these three sub-drainage systems are Stations YC4, YC9, and YC11. Since the observations were performed irregularly at the Station YC9, the water level information at this station was not considered in this research. Therefore, the input variables for the water level prediction at Station YC10 were the mean areal precipitations and the water level information of Stations YC4, YC10, and YC11. The learning target of the RNN model can be referred to the water level observations of YC10. Four identical RNN structures, each with a single output, were designed for 5-, 10-, 15-, and 20-min-ahead water level predictions. The inputoutput combination of RNN models can be represented as follows.

$$
\begin{array}{r}
\hat{L}_{\mathrm{YC} 10}(t+n)=f\left(L_{\mathrm{YC} 4}(t), L_{\mathrm{YC} 10}(t), L_{\mathrm{YC} 11}(t), P(t)\right) \\
n \in 5,10,15,20 .
\end{array}
$$




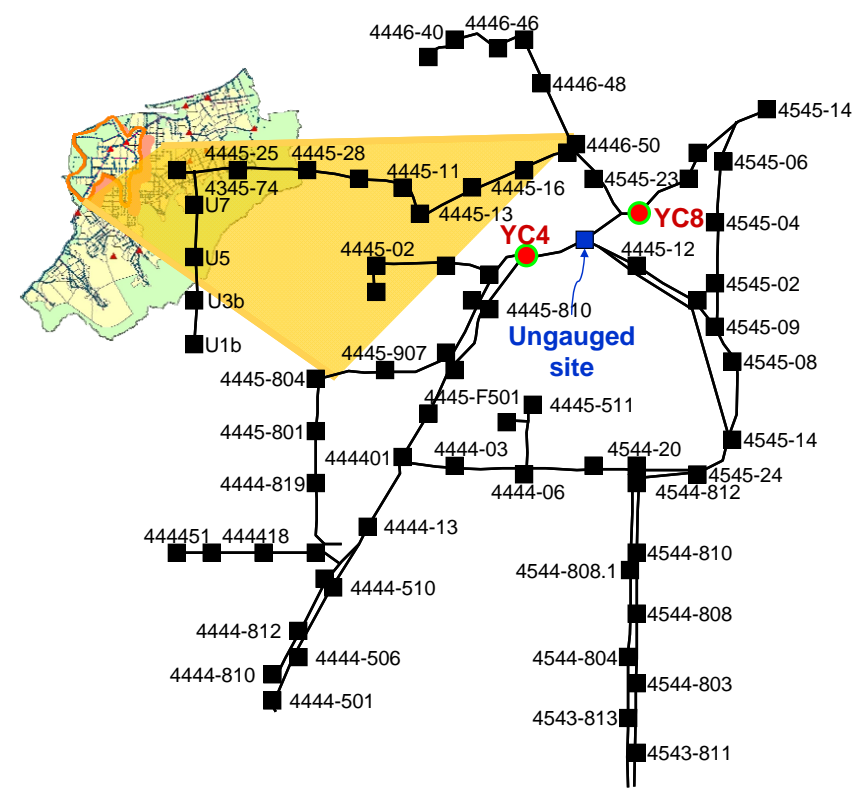

Fig. 3. The location of the ungauged site.

where $\hat{L}(t+n)$ is the model forecast at lead time of $n$ minutes, $L(t)$ is the observed water level at time $t$, and $P(t)$ is the observed precipitation at time $t$.

The time step is set as $5 \mathrm{~min}$ because the operational time step of Yu-Cheng pumping station is 5 min. Owing to current operating procedure of pumps is highly dependent on the experience of local operators who require accurate information on water level prediction for next few steps. The time of concentration of this catchment is about $70 \mathrm{~min}$. There is a trade-off between the accuracy and the lead time of model predictions. To ensure good accuracy of water level prediction, the outputs of the constructed model are designed for a lead time up to $20 \mathrm{~min}$ which is required by local operators. Twenty minutes is enough for reliable and effective operation of pumps to drain runoff water away and reduce the risk of flooding if the water level prediction is precise.

Moreover, in consideration of the historical inundation events recorded by the Hydraulic Engineering Office of Taipei City Government, we selected an ungauged site, located between Stations YC4 and YC8, and marked it with a blue square as shown in Fig. 3. In other words, this selected ungauged site is prone to inundation. Each number displayed in Fig. 3 indicated a manhole related to a drain. Before the construction of a water level prediction model at an ungauged site, it should be confirmed if the quality of the synthetic data of the water levels is appropriate or not. That is to say, the errors may propagate to the RNN if there are large biases in the synthetic data generated by the SWMM. Hence, it is necessary to verify the applicability of a SWMM to the conditions of an urban sewerage system. In this research, the parameters of the SWMM were calibrated first on the historical gauge measurements basis. Then, the flows simulated

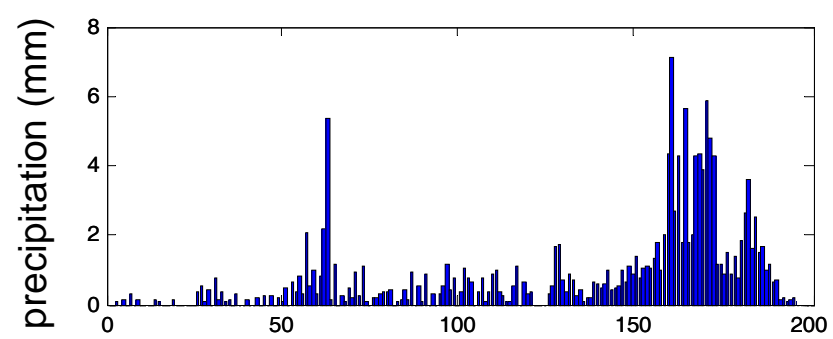

(a)

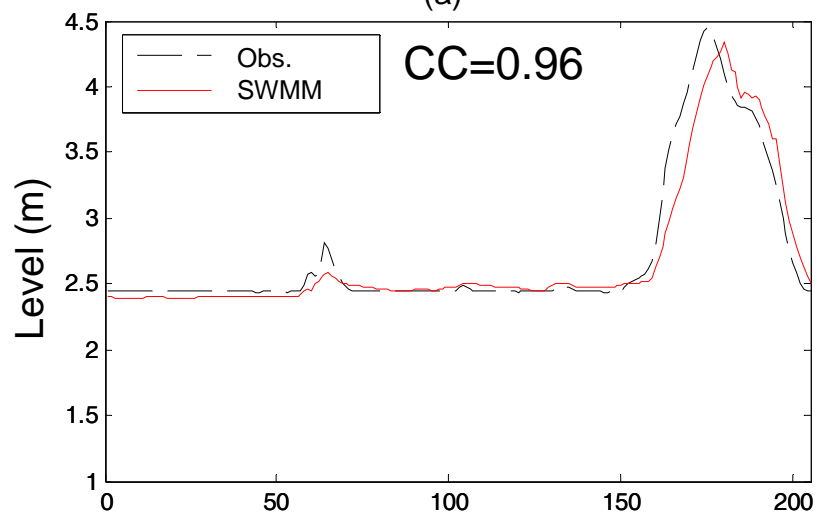

(b)

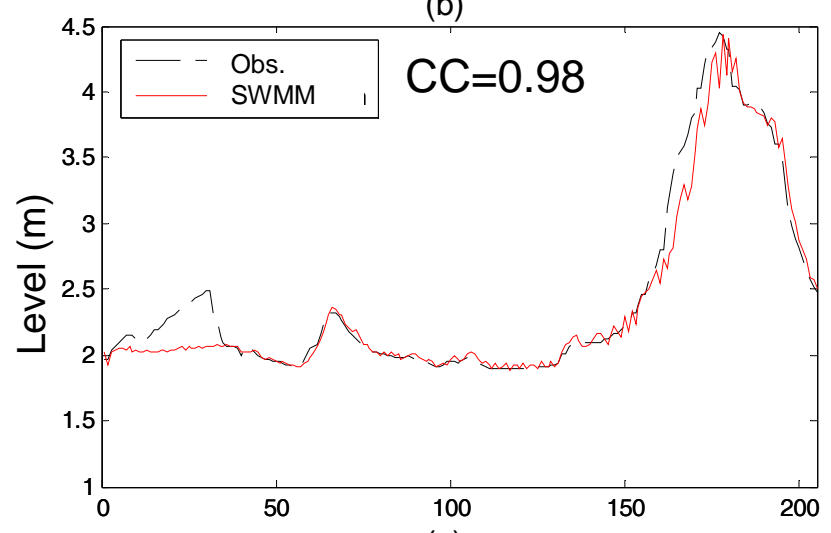

(c)

Time (5-min)

Fig. 4. (a) The rainfall hyetograph, (b) the SWMM hydrograph at Station YC4, (c) the SWMM hydrograph at Station YC8.

by the SWMM corresponding to Station YC4 and Station YC8 were evaluated. Figure 4a showed the rainfall hyetograph of an event randomly selected from datasets, whereas Fig. $4 \mathrm{~b}$ and $\mathrm{c}$ showed the hydrographs of the SWMM output values versus the observation values at Stations YC4 and YC8, accordingly. It clearly indicated that the water levels estimated by the SWMM well captured the main trends of observations at both stations. The accuracies of the simulated flows at Stations YC4 and YC8 were 0.96 and 0.98 each in terms of the correlation coefficient (CC). Results displayed in Fig. 4 confirmed a high reliability of the SWMM simulations. Frankly speaking, the calibration procedure of SWMM is complex, for example, the optimization of parameters usually takes time and have to be calibrated event by event in 
order to obtain accurate estimation of water level. Undoubtedly, a well calibrated SWMM is able to generate suitable estimation of water level and can be applied to any ungauged site if estimation is required. Because the future rainfall pattern is not available at current time, the SWMM could only be used for estimation, not for prediction. Consequently, the RNN is constructed to make the forecasting. Therefore, the advantages of have both SWMM and RNN in this study are (1) to use SWMM to generate data at specific ungauged site as the learning target of RNN; (2) to use RNN fed with the SWMM outputs as learning targets for the purpose of water level prediction at an ungauged site.

For predicting the water level at the selected ungauged site, the input variables of a RNN consists of the precipitations and the water level information of the nearby upstream and downstream stations; namely, the mean areal precipitations and water level observation values at Stations YC4 and YC8. It should be noticed that the learning target values to the RNN is replaced by the synthetic data, assumed as the observation values, of water levels generated from the SWMM at the ungauged site. The RNN network structure is expressed in Fig. 5 and its input-output combination for water level prediction at ungauged site can be represented as follows.

$\hat{L}_{u}(t+n)=f\left(L_{\mathrm{YC} 4}(t), L_{\mathrm{YC} 8}(t), P(t)\right) \quad n \in 5,10,15,20$.

where $\hat{L}_{u}(t+n)$ is the water level prediction at ungauged site at lead time of $n$ minutes, $L_{\mathrm{YC} 4}(t)$ and $L_{\mathrm{YC} 8}(t)$ is the observed water level of Stations YC4 and YC8 at time $t$, and $P(t)$ is the observed precipitation at time $t$.

The best of this technique is this model can produce the water level predictions at any specific location no matter whether water level measurements are available or not. It means this water level prediction model can be extended to any ungauged sites if the observation values of its nearby rainfalls and water levels are available. The RNN learned from both external, Stations YC4, YC8, and P, and internal, the feedback links, input information and further optimized its parameters in accordance with the errors calculated from related outputs and synthetic data. As far as the model optimization procedure is concerned, the number of hidden neurons was first decided (three hidden neurons) by using the trial-and-error method after the determination of input dimension. Second, a tolerable error was set to 0.0001 and the number of iteration was set to 500 because the learning algorithm used herein was the gradient descent method which has the characteristic of fast convergence. Such training process is usually no more than $5 \mathrm{~min}$ and is repeated for 50 times to find appropriate initial values. Consequently, the time spent for optimizing the parameters is about $4 \mathrm{~h}$.

Three error statistics are chosen to assess the consistency between the water level monitoring records and the RNNbased predictions; that is, the correlation coefficient (CC), coefficient of efficiency (CE), and normalized root-meansquare error (NRMSE). All of these indices are widely used

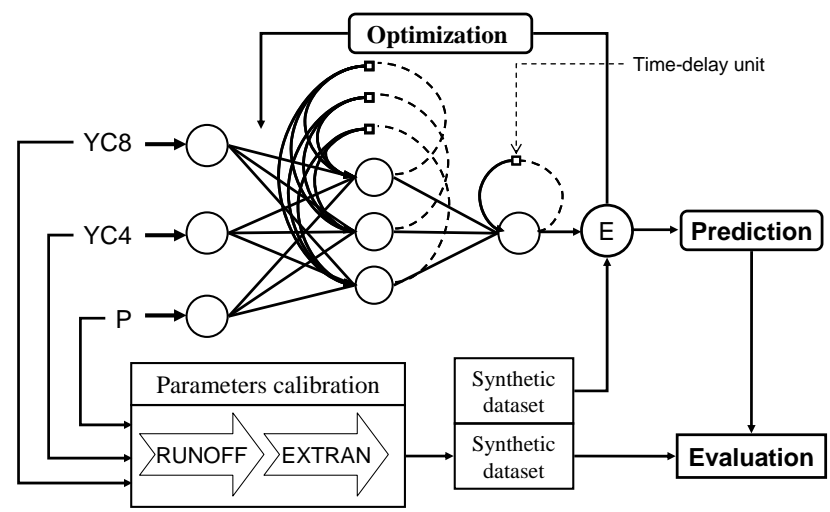

Fig. 5. The architecture of a RNN to predict the water level at the ungauged station.

to estimate the fitness to the hydrological models in hydrological applications, and moreover to facilitate the comparison of different estimated/predicted results. The three criteria are defined as follows:

$\mathrm{CC}=\frac{\sum_{i=1}^{N}(Q(i)-\bar{Q})(\hat{Q}(i)-\overline{\hat{Q}})}{\sqrt{\sum_{i=1}^{N}(Q(i)-\bar{Q})^{2} \sum_{i=1}^{N}(\hat{Q}(i)-\overline{\hat{Q}})^{2}}}$

$\mathrm{CE}=1-\frac{\sum_{i=1}^{N}(Q(i)-\hat{Q})^{2}}{\sum_{i=1}^{N}(Q(i)-\bar{Q})^{2}}$

$\mathrm{NRMSE}=\frac{1}{\sigma} \sqrt{\frac{\sum_{i=1}^{N}(\hat{Q}(i)-Q(i))^{2}}{N}}$

where $\hat{Q}$ is the forecasted water level $(m)$ and $Q$ is the observed water level $(m) ; \bar{Q}$ and $\overline{\hat{Q}}$ are the means of the water levels associated with observation values and forecast values, respectively. $\sigma$ is the standard deviation of the observation values.

\section{Results and discussion}

\subsection{Performance of water level predictions at the Gauging Station YC10}

Table 2 shows the results obtained from the RNN for the water level predictions at Station YC10. The results implied that the model was well trained with a consistent performance and therefore produced precise testing results for multi-stepahead forecasts. The testing performances of the water level predictions of 5-, 10-, 15-, and 20-min-ahead are rather good. 
Table 2. Results obtained from the RNN for water level prediction at Station YC10.

\begin{tabular}{cccccc}
\hline \multirow{6}{*}{ Index } & \multicolumn{4}{c}{ Lead time } \\
\cline { 3 - 6 } & & $5 \mathrm{~min}$ & $10 \mathrm{~min}$ & $15 \mathrm{~min}$ & $20 \mathrm{~min}$ \\
\hline \multirow{4}{*}{ Training } & $\mathrm{CC}$ & 0.99 & 0.99 & 0.99 & 0.99 \\
& $\mathrm{CE}$ & 0.99 & 0.99 & 0.98 & 0.97 \\
& NRMSE & 0.08 & 0.08 & 0.15 & 0.16 \\
\hline \multirow{4}{*}{ Validation } & $\mathrm{CC}$ & 0.99 & 0.99 & 0.97 & 0.97 \\
& $\mathrm{CE}$ & 0.99 & 0.98 & 0.95 & 0.93 \\
& NRMSE & 0.12 & 0.16 & 0.23 & 0.26 \\
\hline \multirow{5}{*}{ Testing } & $\mathrm{CC}$ & 0.99 & 0.99 & 0.97 & 0.97 \\
& $\mathrm{CE}$ & 0.99 & 0.99 & 0.97 & 0.95 \\
& NRMSE & 0.11 & 0.11 & 0.22 & 0.26 \\
\hline
\end{tabular}

(a)
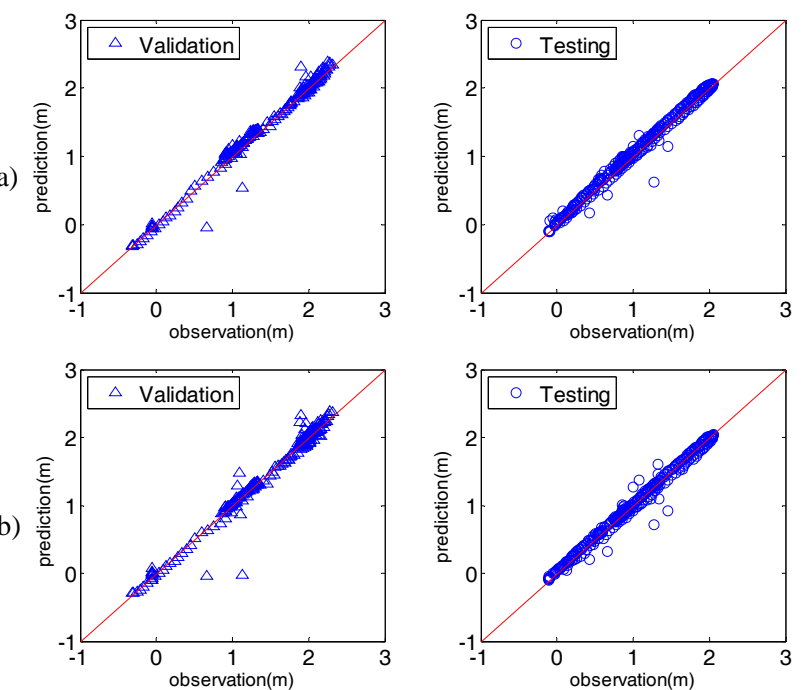

(c)
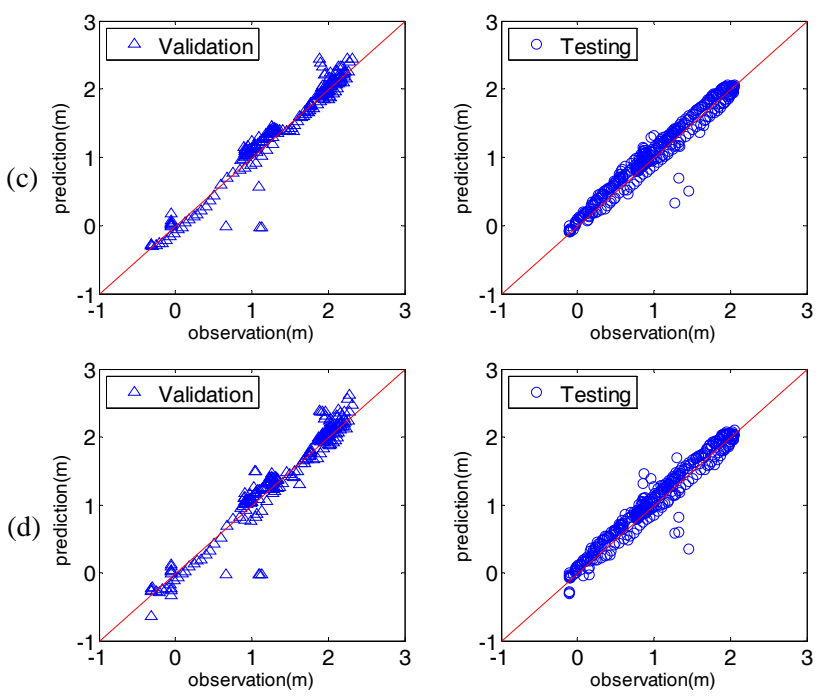

Fig. 6. The scattering plots of the RNN outputs versus the observations for (a) 5-, (b) 10-, (c) 15-, and (d) 20-min-ahead predictions in both validation and testing sets.

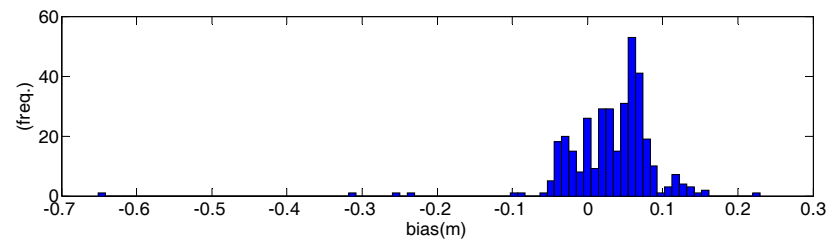

(a)

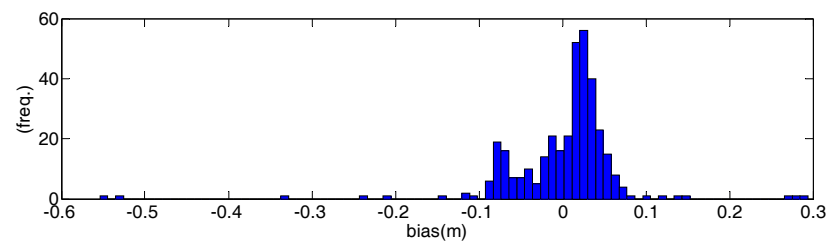

(b)

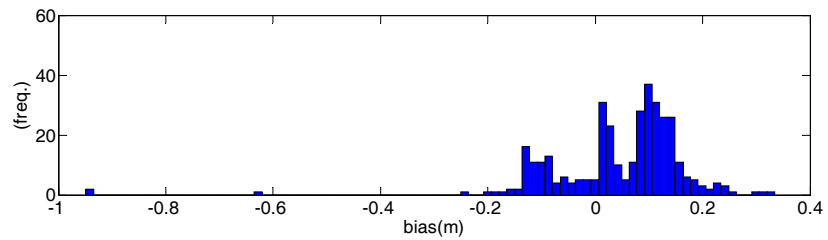

(c)

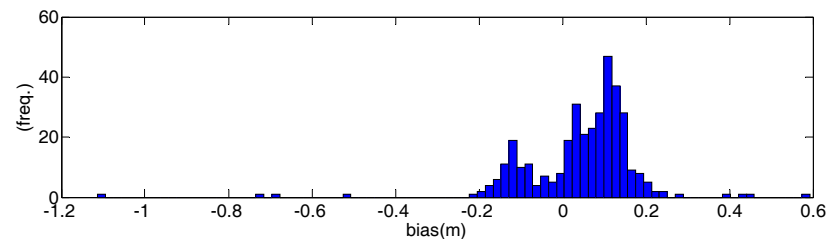

(d)

Fig. 7. The error distribution of RNN outputs in testing phase for (a) 5-, (b) 10-, (c) 15-, and (d) 20-min-ahead predictions.

We feel confident that the RNN-based water level prediction model is capable of capturing the major trends of observations with accuracy higher than 0.97 of correlation coefficient, see Table 2. In terms of coefficient of efficiency (CE), the consistency between the water level observation values and the prediction values indicates that the RNN has the ability to predict highly nonlinear and variable systems, such as urban drainage/sewerage systems. Figure 6 illustrates the scattering plots of the RNN outputs in both validation and testing sets versus the observations for 5 to 20 -min-ahead predictions. The results also prove that the RNN can be well trained for learning any input-output relations if sufficient data can be provided.

Figure 7 shows the error distribution of RNN outputs in testing phase for 5 to 20 -min-ahead predictions. In the 5and 10-min-ahead predictions of the model, the biases between the outputs and observation values are mostly within $10 \mathrm{~cm}$ except for the connection between a low water level event and a high level event with a slight underestimation. A similar phenomenon also occurs in the 15- and 20-minahead predictions since the water level measurements rise 
Table 3. Testing results of the water level prediction at an ungauged site.

\begin{tabular}{lccc}
\hline & CC & CE & NRMSE \\
\hline 5-min-ahead & 0.98 & 0.83 & 0.36 \\
10-min-ahead & 0.97 & 0.82 & 0.42 \\
15-min-ahead & 0.97 & 0.76 & 0.43 \\
20-min-ahead & 0.95 & 0.74 & 0.44 \\
\hline
\end{tabular}

rapidly from $0.1 \mathrm{~m}$ to $1.27 \mathrm{~m}$ within $20 \mathrm{~min}$. However, there is no precipitation measured during this period. One possible explanation may be the failure of hardware instruments. In summary, the RNN model proved its ability to make precise predictions of 20-min-ahead water levels and performed well for peak flow predictions.

\subsection{Performance of water level predictions at an ungauged site}

As mentioned previously, there is a need for building the water level prediction at an ungauged site since monitoring the water levels in urban sewerage systems is not yet very common in Taiwan. In order to conquer the difficulty in predicting the water level at an ungauged site, we utilized the precipitation and the water level information of the nearby stations as input and the synthetic data obtained form the SWMM as the target values, because of the unavailability of real observation values at this site, to train the RNN. Table 3 shows the testing results calculated by all criteria at the chosen ungauged site. For 5- to 20-min-ahead predictions, the values of $\mathrm{CC}$ are higher than 0.95 , indicating that the RNN can effectively predict the water level hydrographs even at an ungauged site. Nevertheless, values of CE, falling between 0.74 and 0.83 , are relatively low which implies that the RNN slightly underestimated some peak flows with an error percentage under $10 \%$. Figure 8 illustrates the scattering plots of the RNN outputs versus the SWMM estimations. For the 5-min-ahead prediction, refer to Fig. 8a, the prediction values are very close to the ideal line which indicates a high accuracy of the RNN outputs. In the analysis of the 10to 20 -min-ahead prediction values, see Fig. $8 \mathrm{~b}-\mathrm{d}$, it is quite obvious that the RNN underestimates some observation values. The major reason for producing such underestimation is due to the time-lag problems which occur in the output process of the RNN. Fortunately, the phenomenon of such underestimation is not that serious. In summary, the results obtained from RNN are still acceptable, indicating that the RNN is also applicable to the water level predictions at ungauged sites by using synthetic data sets generated from a well calibrated SWMM.

Overall, the study demonstrates that the RNN model is capable of learning the time-varying processes in urban sewerage systems, and therefore providing precise water level

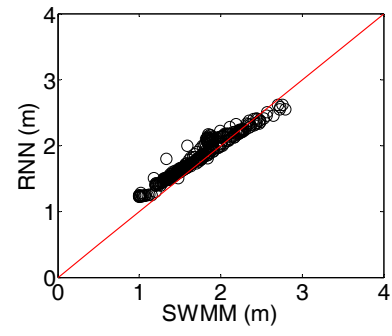

(a)

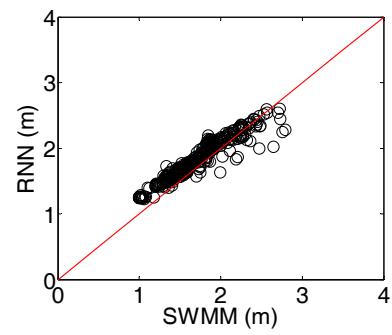

(c)

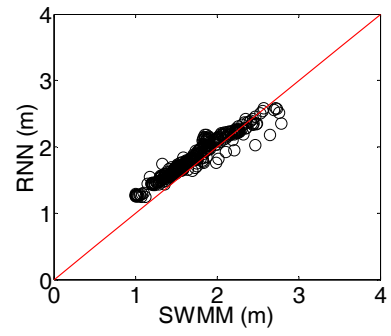

(b)

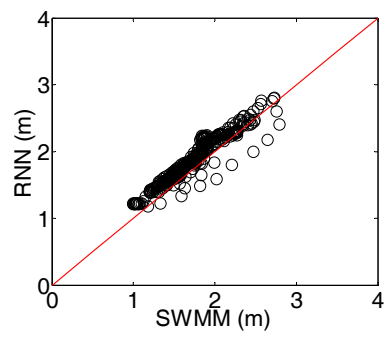

(d)
Fig. 8. The scattering plots of the RNN outputs versus the SWMM estimations for (a) 5-, (b) 10-, (c) 15-, and (d) 20-min-ahead predictions in testing set.

predictions up to $20 \mathrm{~min}$ ahead. It is interesting to inspect the model performance at gauged and ungauged sites. For example, the RNN models produced smaller predictive error at gauged site but larger predictive error at ungauged site (in terms of CE and NRMSE) even though the correlation coefficients at both sites are higher than 0.95 . This is because the hydrograph of the synthetic data generated by SWMM is not as smooth as that of observations (see Fig. 4). In other words, the bias produced from synthetic data does propagate to RNN model, and therefore resulted in larger predictive error. Besides, the correlation coefficients of the SWMM simulations achieved 0.96 and 0.98 at YC4 and YC8, respectively, which indicates the best performance obtained at ungauged site will not be higher than 0.98 . Our results conform to this limitation (see Table 3) and demonstrate that the predictive capabilities of RNN models are similar no matter where it is.

Regarding the application possibility of the constructed model, it depends on both model reliability and stability. In this study, the results obtained from RNN are precise and show that the reliability of the constructed RNN is qualified to be applied to the water level predictions at urban sewerage systems. As for model stability, it can be demonstrated (Table 2) that the model was well constructed using a considerable amount of data during training phase. Meanwhile, the second independent dataset was used to optimize the structure and connected weights in the validation phase. The function of validation data is to prevent the model from being over-trained and to efficiently increase the model's generalization capability. Results obtained from RNN strongly revealed that the model has optimized and stabilized in terms of 
highly accurate and consistent performance in both training and validation phases. Once the model was well trained and validated, it would be able to make on-line forecast instantaneously and recursively for the coming storm event. Therefore, we then make recursive forecast by using the testing dataset. The testing results produced by RNN were comparable to those of training and validation which means the stability of RNN is robust. Overall, we believe that the built RNN model can be applied and perform well in practice.

\section{Conclusions}

In this research, the recurrent-neural-network-based hydrological models have been developed to predict the water levels at both gauged and ungauged sites. This technique was applied to a sewerage system in the biggest urbanized catchment in Taipei City. Eight RNN models (four for YC10 gauged site, and four for ungauged site), each with a single output, were calibrated by using the mean areal precipitations and upstream hydrological information, and then built for the multi-step-ahead water level predictions. The related outputs can provide important prior knowledge and/or information for the successful operation of pumping stations in an urban sewerage system, and the accurate water level predictions should offer an improvement in preventing a city from inundation.

The RNN-based hydrological models can precisely predict the observation values at the catchment outlet. For the prediction at the gauged site, Station YC10, the RNN produced excellent water level outputs for 5- up to 20-min-ahead predictions. As far as the statistical criteria are concerned, the values of both $\mathrm{CC}$ and $\mathrm{CE}$ are higher than 0.97 in testing phase, indicating that the RNN is very suitable to model the nonlinear and time-varying mechanisms of an urban sewerage system. For the prediction at the ungauged site, the SWMM was used for computing storm sewer flow and generating data at the selected manhole. Based on the evaluation of the SWMM's applicability to an urban sewerage system, the synthetic data at an ungauged manhole were generated for the training of the RNN. These results pointed out that even though the forecasts of the RNN slightly underestimated the peak flows, the RNN model effectively captured the major trends of the water level hydrographs produced by the SWMM. This paper provided an applicable way to predict water level at ungauged site and the preliminary investigation showed that the result of prediction obtained from RNN trained with data generated from SWMM is reliable. We are certain that this proposed method can be used in a more flexible way for constructing the hydrological prediction model at any specific manhole. Likewise, the results can be of great help to verify the inundation risk and to enhance the efficiency of a pumping station in an inundation-prone area if a suitable operational strategy can be made.
Acknowledgements. This research was sponsored by the Water Resource Agency, Taipei, Taiwan. The Taipei City Government provided the sewerage gauging data for analysis purpose in this research.

Edited by: E. Toth

\section{References}

Ackerman, D. and Schiff, K.: Modeling storm water mass emissions to the southern California bight, J. Environ. Eng-Asce, 129(4), 308-317, 2003.

Akhtar, M. K., Corzo, G. A., van Andel, S. J., and Jonoski, A.: River flow forecasting with artificial neural networks using satellite observed precipitation pre-processed with flow length and travel time information: case study of the Ganges river basin, Hydrol. Earth Syst. Sci., 13, 1607-1618, doi:10.5194/hess-131607-2009, 2009.

Baffaut, C. and Delleur, J. W.: Calibration of Swmm Runoff Quality Model with Expert System, J. Water Res. Pl-Asce, 116(2), 247261, 1990.

Besaw, L. E., Rizzo, D. M., Bierman, P. R., and Hackett, W. R.: Advances in ungauged streamflow prediction using artificial neural networks, J. Hydrol., 386(1-4), 27-37, 2010.

Borah, D. K. and Bera, M.: Watershed-scale hydrologic and nonpoint-source pollution models: Review of mathematical bases, T. Asae, 46(6), 1553-1566, 2003.

Bruen, M. and Yang, J. Q.: Combined hydraulic and black-box models for flood forecasting in urban drainage systems, J. Hydrol. Eng., 11(6), 589-596, 2006.

Chandramouli, V. and Deka, P.: Neural network based decision support model for optimal reservoir operation, Water Resour. Manag., 19(4), 447-464, 2005.

Chang, F. J., Chang, L. C., and Huang, H. L: Real-time recurrent learning neural network for stream-flow forecasting, Hydrol. Process., 16(13), 2577-2588, 2002.

Chang, F. J. and Chang, Y. T.: Adaptive neuro-fuzzy inference system for prediction of water level in reservoir, Adv. Water Resour., 29(1), 1-10, 2006.

Chang, L. C. and Chang, F. J.: Intelligent control for modelling of real-time reservoir operation, Hydrol. Process., 15(9), 16211634, 2001.

Chang, L. C., Shen, H. Y., Wang, Y. F., Huang, J. Y., and Lin, Y. T. Clustering-based hybrid inundation model for forecasting flood inundation depths, J. Hydrol., 385(1-4), 257-268, 2010.

Chaves, P. and Chang, F. J.: Intelligent reservoir operation system based on evolving artificial neural networks, Adv. Water Resour., 31(6), 926-936, 2008.

Chiang, Y. M. and Chang, F. J.: Integrating hydrometeorological information for rainfall-runoff modelling by artificial neural networks, Hydrol. Process., 23(11), 1650-1659, 2009.

Chiang, Y. M., Chang, F. J., Jou, B. J. D., and Lin, P. F.: Dynamic ANN for precipitation estimation and forecasting from radar observations, J. Hydrol., 334(1-2), 250-261, 2007a.

Chiang, Y. M., Hsu, K. L., Chang, F. J., Hong, Y., and Sorooshian, S.: Merging multiple precipitation sources for flash flood forecasting, J. Hydrol., 340(3-4), 183-196, 2007 b.

Chiang, Y. M., Chang, L. C., and Chang, F. J.: Comparison of staticfeedforward and dynamic-feedback neural networks for rainfall- 
runoff modeling, J. Hydrol., 290(3-4), 297-311, 2004.

Coulibaly, P. and Baldwin, C. K.: Nonstationary hydrological time series forecasting using nonlinear dynamic methods, J. Hydrol., 307(1-4), 164-174, 2005.

Coulibaly, P. and Evora, N. D.: Comparison of neural network methods for infilling missing daily weather records, J. Hydrol., 341(1-2), 27-41, 2007.

Denault, C., Millar, R. G., and Lence, B. J.:Assessment of possible impacts of climate change in an urban catchment, J. AM. Water Resour. As., 42(3), 685-697, 2006.

Huber, W. C. and Dickinson, R. E.: Strom water management model, User's Manual Ver. IV, US Environmental Protection Agency, 1988.

Hung, N. Q., Babel, M. S., Weesakul, S., and Tripathi, N. K.: An artificial neural network model for rainfall forecasting in Bangkok, Thailand, Hydrol. Earth Syst. Sci., 13, 1413-1425, doi:10.5194/hess-13-1413-2009, 2009.

Kumar, D. N., Raju, K. S., and Sathish, T.: River flow forecasting using recurrent neural networks, Water Resour. Manag., 18(2), 143-161, 2004.

Loke, E., Warnaars, E. A., Jacobsen, P., Nelen, F., and Almeida, M. D.: Artificial neural networks as a tool in urban storm drainage, Water Sci. Technol., 36(8-9), 101-109, 1997.

Maharjan, M., Pathirana, A., Gersonius, B., and Vairavamoorthy, K.: Staged cost optimization of urban storm drainage systems based on hydraulic performance in a changing environment, Hydrol. Earth Syst. Sci., 13, 481-489, doi:10.5194/hess-13-4812009, 2009.
Maity, R. and Kumar, D. N.: Basin-scale stream-flow forecasting using the information of large-scale atmospheric circulation phenomena, Hydrol. Process., 22(5), 643-650, 2008.

Mishra, A. K. and Desai, V. R.: Drought forecasting using feedforward recursive neural network, Ecol. Model., 198(1-2), 127138, 2006.

Nasseri, M., Asghari, K., and Abedini, M. J.: Optimized scenario for rainfall forecasting using genetic algorithm coupled with artificial neural network, Expert Syst. Appl., 35(3), 1415-1421, 2008.

Razavi, S. and Karamouz, M.: Adaptive neural networks for flood routing in river systems, Water Int., 32(3), 360-375, 2007.

Sahoo, G. B., Schladow, S. G., and Reuter, J. E.: Forecasting stream water temperature using regression analysis, artificial neural network, and chaotic non-linear dynamic models, J. Hydrol., 378(34), 25-342, 2009.

Sudheer, K. P., Srinivasan, K., Neelakantan, T. R., and Srinivas, V. V.: A nonlinear data-driven model for synthetic generation of annual streamflows, Hydrol. Process., 22(12), 1831-1845, 2008.

Toth, E.: Classification of hydro-meteorological conditions and multiple artificial neural networks for streamflow forecasting, Hydrol. Earth Syst. Sci., 13, 1555-1566, doi:10.5194/hess-131555-2009, 2009.

Tsihrintzis, V. A. and Hamid, R.: Runoff quality prediction from small urban catchments using SWMM, Hydrol. Process., 12(2), 311-329, 1998.

Yazdani, M. R., Saghafian, B., Mahdian, M. H., and Soltani, S.: Monthly Runoff Estimation Using Artificial Neural Networks, J. Agri. Sci. Technol., 11(3), 355-362, 2009. 